\title{
An interactive approach to the stochastic multiobjective allocation problem
}

\author{
Maciej Nowak ${ }^{1, *}$ and Tadeusz Trzaskalik ${ }^{1}$ \\ ${ }^{1}$ Department of Operations Research, University of Economics in Katowice \\ ul. 1 Maja 50, 40-287 Katowice, Poland \\ E-mail: 〈\{maciej.nowak, tadeusz.trzaskalik\}@ue.katowice.pl〉
}

\begin{abstract}
The paper considers the stochastic multiobjective allocation problem. An assumption is made that a particular resource should be allocated to $T$ projects. The specified level of each goal (along with the known probabilities) can be obtained based on the amount of the resources allocated. We will propose an identification strategy procedure that the decision maker can implement. Our technique combines multiobjective dynamic programming and an interactive approach. First, efficient strategies are identified using Bellman's principle applied to the multiobjective problem. Next, a dialog procedure is used to find a solution that is satisfactory for the decision maker. A numerical example is presented to show how the procedure can be applied.
\end{abstract}

Key words: stochastic allocation problem, multiobjective dynamic programming, interactive approach, stochastic dominance

Received: October 4, 2014; accepted: March 19, 2015; available online: March 30, 2015

DOI: $10.17535 /$ crorr.2015.0023

\section{Introduction}

The stochastic multiobjective allocation problem (SMAP), considered in this paper, can be described in the following manner. A company has a limited amount of a particular resource that can be allocated to various projects. We assume that the decision maker has defined $K$ goals that are to be achieved. Several levels of achievement are specified for each goal. The issue becomes a stochastic problem, i.e. a particular amount of the resource is allocated to a certain project, and a planned level of the goal is achieved at a certain probability. Hence, a decision has to be made as to which projects to implement and the intensity of their realization.

The single objective deterministic allocation problem, which was formulated above, are described and solved in [1] and [2]. The dynamic programming

${ }^{*}$ Corresponding author. 
approach was applied. A biobjective deterministic allocation problem is analyzed in [10]. The manner in which the vector version of Bellman's optimality principle can be used to identify the whole set of non-dominated solutions has also been shown.

Various other types of stochastic allocation problems have been considered in literature. For example, sensor allocation management and dynamic programming for the stochastic resource allocation problem was considered in [4]. Techniques in finding an approximate solution for a certain class of resource allocation problems that arise in stochastic sequential decision-making problems and are computationally efficient were considered in [3]. The problem of allocating a quantity of a resource to several activities to satisfy stochastic demands occurring randomly is described in [6]. An application of dynamic resource allocation in wireless communication using stochastic optimization is described in [5].

Our goal is to propose a procedure to solve SMAP, which combines multiobjective dynamic programming and an interactive approach. We will identify a strategy to be implemented by a decision maker, and which suits best the decision maker's preferences. The proposed procedure is a modification of the procedure described in [7] and [9], and has been adapted to the problem at hand. First, a set of efficient strategies is identified using Bellman's principle of optimality, which has been adapted to the multiobjective problem. Next, an interactive procedure is used to find the final solution. In each iteration, a candidate strategy is presented to the decision maker. If satisfied with the proposal, the procedure ends; otherwise, the decision maker is asked for preferences by specifying the criterion values to be achieved, or at least indicating the criterion that is to be improved.

The rest of the paper is organized into a number of sections. Section 2 presents a stochastic allocation problem is presented as an example of dynamic programming with partially ordered criteria space. Next, Section 3 describes the proposed interactive procedure for identifying the final solution. Section 4 presents a numerical example. Finally, the last section provides some final remarks.

\section{The stochastic allocation problem as an example of dynamic programming with partially ordered criteria space}

A partially ordered criteria space $(\mathbf{W}, \leq, \circ)$ consists of the set $\mathbf{W}$, the preference relation $\leq$ ( "not worse than") and the binary operator $\circ$.

In describing a discrete decision process, we identify sets of feasible states and decisions, transition functions, period criteria functions and multi-period criteria functions. By applying Bellman's principle of optimality, the set of nondominated vectors of evaluations in the criteria space and efficient process 
realizations in the decision space can be identified. The details are described in $[9,11]$.

Let us consider the set of probability sequences, defined in the following way [13]:

$$
\mathbf{W}=\left\{\left(p_{0}, p_{1}, \ldots, p_{n}\right): n \in \mathbf{N}, p_{n}>0, p_{i} \geq 0, \sum_{i=0}^{n} p_{i}=1\right\}
$$

where $p_{i}=\mathrm{P}(X=i), n=\max \left\{k \in \mathbf{N}, p_{k}>0\right\}$. For the sake of simplicity, we assume that random variables take only nonnegative integer values. This is an example of a partially ordered criteria space.

Let $p, q \in \mathbf{W}, p=\left(p_{1}, \ldots, p_{n}\right), q=\left(q_{1}, \ldots, q_{m}\right)$. We define the operator $\circ$ as follows:

$$
p \circ q=\left(r_{0}, r_{1}, \ldots, r_{n+m}\right)
$$

where $r_{i}=\sum_{\substack{k, l \\ k+l=i}} p_{k} q_{l}$ for $i=\overline{1, n+m}$.

To define the relation $\leq$ we use FSD (First Stochastic Dominance) and SSD (Second Stochastic Dominance) relations:

where:

$$
p \leq q \Leftrightarrow q \operatorname{FSD} p \quad \vee \quad q \operatorname{SSD} p
$$

$$
\begin{gathered}
q \operatorname{FSD} p \Leftrightarrow \forall_{i \in \overline{0, n}} \sum_{k=0}^{i} q_{k} \leq \sum_{k=0}^{i} p_{k} \\
q \operatorname{SSD} p \Leftrightarrow \quad \forall_{i \in \overline{0, n}} \sum_{k=0}^{i} \sum_{l=0}^{k} q_{l} \leq \sum_{k=0}^{i} \sum_{l=0}^{k} p_{l}
\end{gathered}
$$

The stochastic allocation problem considered in this paper can be regarded as a $T$-stage decision problem in a partially ordered criteria space. The number of stages is determined by the number of analyzed projects. In stage $t$ ( $t \in \overline{1, T}$ ), the decision on the number of units allocated to a particular project is made.

The set $\mathbf{Y}_{t}$ of feasible states $y_{t}$ in the consecutive stages $t \in \overline{1, T}$ is defined as follows:

$$
\mathbf{Y}_{t}=\left\{y_{t} \in \mathbf{N}^{0}: 0 \leq y_{t} \leq n_{0}\right\}
$$

The set of feasible decisions for the consecutive states $y_{t} \in \mathbf{Y}_{t}$ for $t \in \overline{1, T}$ is defined in the following way:

$$
\mathbf{X}_{t}\left(y_{t}\right)=\left\{x_{t} \in \mathbf{N}^{0}: 0 \leq x_{t} \leq y_{t}\right\}
$$

For $t \in \overline{1, T}$, the transition functions are defined as follows: 


$$
y_{t+1}=y_{t}-x_{t}
$$

Each of the $K$ criteria functions considered is an element of the set $\mathbf{W}$, described by formula (1). The criteria space $\mathbf{W}^{K}$ is the product of $K$ structures W. The operator $\circ^{K}$ and relation $\leq^{K}$ are defined as follows:

$$
\begin{gathered}
\left(p^{1}, p^{2}, \ldots, p^{K}\right) \circ^{K}\left(q^{1}, q^{2}, \ldots, q^{K}\right) \stackrel{\text { def }}{=}\left(p^{1} \circ q^{1}, p^{2} \circ q^{2}, \ldots, p^{K} \circ q^{K}\right) \\
\left(p^{1}, p^{2}, \ldots, p^{K}\right) \leq^{K}\left(q^{1}, q^{2}, \ldots, q^{K}\right) \stackrel{\text { def }}{=} p^{1} \leq q^{1} \wedge p^{2} \leq q^{2} \wedge \ldots \wedge p^{K} \leq q^{K}
\end{gathered}
$$

The relation $\leq^{K}$ holds if FSD or SSD relations hold for each criterion [12].

\section{The proposed interactive procedure}

Interactive techniques are widely used for solving multiobjective problems. In this approach, the preference information is obtained in a stepwise manner. The decision maker is asked to define which criteria influence his or her preferences and to provide preference information for a given solution or set of solutions (local preference information).

Various interactive techniques were proposed for the discrete stochastic decision making problems by one of the authors of this paper [8]. A modification of the INDECM technique is presented [7]. The problem is solved iteratively. The decision maker analyzes the results in each iteration and decides how to improve the current solution. The procedure is continued until a satisfactory solution is identified.

As the evaluation of each alternative with relative to each criterion is represented by a probability distribution, we must choose what measures to use during the dialog phase of the procedure. These can be either measures of central tendency, or else measures of dispersion. At least one measure must be used for each criterion. The measures should be chosen by the decision maker at the initial phase of the procedure.

The potency matrix is generated in each iteration and presented to the decision maker. It has two rows: the first one groups the worst (pessimistic) values, and the second one, the best (optimistic) values of the considered measures attainable independently within the set of alternatives. The decision maker is asked whether pessimistic values are satisfactory. If the answer is "yes", a request is directed to make the final choice. Otherwise, the decision maker is asked to express preferences by defining values that the measures should achieve (aspiration levels), or at least by indicating the criterion for which the pessimistic value should be improved.

Let $\hat{\mathbf{D}}$ be the set of efficient solutions, $\mathbf{D}^{(t)}$ - the set of solutions analyzed in iteration $l, M$ - the number of measures analyzed, and $\mathbf{G}^{(l)}$ - the potency matrix: 


$$
\mathbf{G}^{(l)}=\left[\begin{array}{lllll}
\underline{g}_{1}^{(l)} & \cdots & \underline{\boldsymbol{g}}_{m}^{(l)} & \cdots & \underline{\boldsymbol{g}}_{M}^{(l)} \\
\overline{\bar{g}}_{1}^{(l)} & \ldots & \overline{\boldsymbol{g}}_{m}^{(l)} & \cdots & \overline{\boldsymbol{g}}_{M}^{(l)}
\end{array}\right]
$$

where: $\underline{g}_{m}^{(l)}$ is the worst value of the $m$-th measure attainable in the set of solutions analyzed in iteration $l$, and $\bar{g}_{m}^{(l)}$ is the best one.

The procedure for identifying the final solution is as follows:

1. Set $l=1, \mathbf{D}^{(1)}=\widehat{\mathbf{D}}$.

2. Identify the potency matrix $\mathbf{G}^{(l)}$.

3. Ask whether the decision maker is satisfied with the pessimistic values. If the answer is "yes", go to (8).

4. Ask whether the decision maker would like to define the aspiration levels for the criteria. If the answer is " $n o$ " - go to (6).

5. Ask the decision maker to specify aspiration levels $\tilde{\boldsymbol{g}}_{m}^{(l)}$ for $m=1, \ldots, M$. Identify the set $\mathbf{D}^{(l+1)}$ which includes the solutions satisfying the decision maker's requirements. If $\mathbf{D}^{(l+1)}=\varnothing$, report this to the decision maker and go to (4), otherwise go to (7).

6. Ask the decision maker to indicate the index $m$ of the measure for which the pessimistic value is unsatisfactory. Identify the set $\mathbf{D}^{(l+1)}$ which groups the solutions for which the value of the $m$-th measure is better than the current pessimistic value $\underline{g}_{m}^{(l)}$.

7. Set $l=l+1$ and go to (2).

8. Ask the decision maker to indicate the index $m$ of the measure that should achieve the optimistic value, select the solution for which the $m$-th measure is equal to the optimistic value $\bar{g}_{m}^{(l)}$.

\section{Numerical example}

The data used in this example are fictitious. The company is going to allocate six units of resources among three projects. Three goals are considered, and three criteria are used to evaluate the level at which the goal is achieved. Tables 1-3 show probabilities that a particular level of goal is achieved for Projects 1, 2 and 3 . 


\begin{tabular}{|c|c|c|c|c|c|c|c|c|c|}
\hline \multirow{2}{*}{$\begin{array}{l}\text { Units of } \\
\text { resource }\end{array}$} & \multirow{2}{*}{ Level } & \multicolumn{3}{|c|}{ Probability } & \multirow{2}{*}{$\begin{array}{l}\text { Units of } \\
\text { resource }\end{array}$} & \multirow{2}{*}{ Level } & \multicolumn{3}{|c|}{ Probability } \\
\hline & & Goal 1 & Goal 2 & Goal 3 & & & Goal 1 & Goal 2 & Goal 3 \\
\hline 0 & 0 & 1.0 & 1.0 & 1.0 & \multirow[t]{2}{*}{4} & 2 & 0.2 & 0.4 & 0.2 \\
\hline \multirow[t]{2}{*}{1} & 0 & 0.5 & 0.3 & 0.8 & & 3 & 0.1 & 0.2 & 0.1 \\
\hline & 1 & 0.5 & 0.7 & 0.2 & \multirow[t]{4}{*}{5} & 0 & 0.2 & 0.1 & 0.4 \\
\hline \multirow[t]{3}{*}{2} & 0 & 0.4 & 0.3 & 0.7 & & 1 & 0.4 & 0.2 & 0.2 \\
\hline & 1 & 0.5 & 0.5 & 0.2 & & 2 & 0.2 & 0.4 & 0.2 \\
\hline & 2 & 0.1 & 0.2 & 0.1 & & 3 & 0.2 & 0.3 & 0.2 \\
\hline \multirow[t]{3}{*}{3} & 0 & 0.3 & 0.2 & 0.6 & \multirow[t]{5}{*}{6} & 0 & 0.1 & 0 & 0.3 \\
\hline & 1 & 0.5 & 0.4 & 0.2 & & 1 & 0.3 & 0.1 & 0.3 \\
\hline & 2 & 0.2 & 0.4 & 0.2 & & 2 & 0.3 & 0.4 & 0.2 \\
\hline \multirow[t]{2}{*}{4} & 0 & 0.2 & 0.1 & 0.5 & & \multirow[t]{2}{*}{3} & \multirow[t]{2}{*}{0.3} & 0.5 & \multirow[t]{2}{*}{0.2} \\
\hline & 1 & 0.5 & 0.3 & 0.2 & & & & & \\
\hline
\end{tabular}

Table 1: Probabilities that a particular level of goal is achieved for Project no. 1

\begin{tabular}{|c|c|c|c|c|c|c|c|c|c|}
\hline \multirow{2}{*}{$\begin{array}{l}\text { Units of } \\
\text { resource }\end{array}$} & \multirow{2}{*}{ Level } & \multicolumn{3}{|c|}{ Probability } & \multirow{2}{*}{$\begin{array}{l}\text { Units of } \\
\text { resource }\end{array}$} & \multirow{2}{*}{ Level } & \multicolumn{3}{|c|}{ Probability } \\
\hline & & Goal 1 & Goal 2 & Goal 3 & & & Goal 1 & Goal 2 & Goal 3 \\
\hline 0 & 0 & 1.0 & 1.0 & 1.0 & \multirow[t]{2}{*}{4} & 2 & 0.4 & 0.3 & 0.2 \\
\hline \multirow[t]{2}{*}{1} & 0 & 0.3 & 0.4 & 0.7 & & 3 & 0.2 & 0.1 & 0.1 \\
\hline & 1 & 0.7 & 0.6 & 0.3 & \multirow[t]{4}{*}{5} & 0 & 0.1 & 0.1 & 0.2 \\
\hline \multirow[t]{3}{*}{2} & 0 & 0.3 & 0.3 & 0.5 & & 1 & 0.2 & 0.2 & 0.3 \\
\hline & 1 & 0.4 & 0.6 & 0.4 & & 2 & 0.5 & 0.4 & 0.3 \\
\hline & 2 & 0.3 & 0.1 & 0.1 & & 3 & 0.2 & 0.3 & 0.2 \\
\hline \multirow[t]{3}{*}{3} & 0 & 0.1 & 0.3 & 0.4 & \multirow[t]{5}{*}{6} & 0 & 0.1 & 0 & 0.2 \\
\hline & 1 & 0.7 & 0.4 & 0.4 & & 1 & 0.3 & 0.2 & 0.2 \\
\hline & 2 & 0.2 & 0.3 & 0.2 & & 2 & 0.3 & 0.4 & 0.4 \\
\hline \multirow[t]{2}{*}{4} & 0 & 0.1 & 0.2 & 0.3 & & 3 & 0.3 & 0.4 & 0.2 \\
\hline & 1 & 0.3 & 0.4 & 0.4 & & & & & \\
\hline
\end{tabular}

Table 2: Probabilities that a particular level of goal is achieved for Project no. 2

\begin{tabular}{|c|c|c|c|c|c|c|c|c|c|}
\hline \multirow{2}{*}{$\begin{array}{l}\text { Units of } \\
\text { resource }\end{array}$} & \multirow{2}{*}{ Level } & \multicolumn{3}{|c|}{ Probability } & \multirow{2}{*}{$\begin{array}{l}\text { Units of } \\
\text { resource }\end{array}$} & \multirow{2}{*}{ Level } & \multicolumn{3}{|c|}{ Probability } \\
\hline & & Goal 1 & Goal 2 & Goal 3 & & & Goal 1 & Goal 2 & Goal 3 \\
\hline 0 & 0 & 1.0 & 1.0 & 1.0 & \multirow[t]{2}{*}{4} & 2 & 0.2 & 0.2 & 0.3 \\
\hline \multirow[t]{2}{*}{1} & 0 & 0.6 & 0.5 & 0.9 & & 3 & 0.1 & 0.2 & 0.1 \\
\hline & 1 & 0.4 & 0.5 & 0.1 & \multirow[t]{4}{*}{5} & 0 & 0.1 & 0.1 & 0.1 \\
\hline \multirow[t]{3}{*}{2} & 0 & 0.2 & 0.4 & 0.7 & & 1 & 0.4 & 0.3 & 0.2 \\
\hline & 1 & 0.3 & 0.5 & 0.2 & & 2 & 0.3 & 0.4 & 0.5 \\
\hline & 2 & 0.5 & 0.1 & 0.1 & & 3 & 0.2 & 0.2 & 0.2 \\
\hline \multirow[t]{3}{*}{3} & 0 & 0.2 & 0.3 & 0.5 & \multirow[t]{4}{*}{6} & 0 & 0.1 & 0.1 & 0.1 \\
\hline & 1 & 0.4 & 0.5 & 0.3 & & 1 & 0.3 & 0.2 & 0.1 \\
\hline & 2 & 0.4 & 0.2 & 0.2 & & 2 & 0.3 & 0.3 & 0.3 \\
\hline 4 & $\begin{array}{l}0 \\
1\end{array}$ & $\begin{array}{l}0.3 \\
0.4\end{array}$ & $\begin{array}{l}0.2 \\
0.4\end{array}$ & $\begin{array}{l}0.3 \\
0.3\end{array}$ & & 3 & 0.3 & 0.4 & 0.5 \\
\hline
\end{tabular}

Table 3: Probabilities that a particular level of goal is achieved for Project no. 3

We are looking for the best allocation of the resource by applying the interactive procedure described above. At the beginning of the first stage, six 
units of the resource are available. Thus, we allocate $0,1,2,3,4,5$ or 6 units for Project 1, hence:

$$
\mathbf{Y}_{1}=\{6\}, \mathbf{X}_{1}(6)=\{0,1,2,3,4,5,6\}
$$

In applying the transition function (8), we obtain the set of feasible states at the beginning of the second stage, which is as follows:

$$
\mathbf{Y}_{2}=\{0,1,2,3,4,5,6\}
$$

If in stage 1 the decision $x_{1}=6$ has been made, then according to the transition function (8) the process will reach the state $y_{2}=0$. As all units of the resource are allocated to project 1 , the only feasible decision in stage 2 is $x_{2}=0$, hence:

$$
\mathbf{X}_{2}(0)=\{0\}
$$

If in stage 1 the decision $x_{1}=5$ has been made, the process will reach the state $y_{2}=1$ at the next stage. In this case, according to (7), two decisions can be made in stage $2: x_{2}=0$ or $x_{2}=1$, hence:

$$
\mathbf{X}_{2}(1)=\{0,1\}
$$

The remaining sets of feasible decisions for the stage 2 are as follows:

$$
\begin{gathered}
\mathbf{X}_{2}(2)=\{0,1,2\}, \mathbf{X}_{2}(3)=\{0,1,2,3\}, \mathbf{X}_{2}(4)=\{0,1,2,3,4\} \\
\mathbf{X}_{2}(5)=\{0,1,2,3,4,5\}, \mathbf{X}_{2}(6)=\{0,1,2,3,4,5,6\}
\end{gathered}
$$

The set of feasible states in stage 3 is the same as in stage 2 :

$$
\mathbf{Y}_{3}=\{0,1,2,3,4,5,6\}
$$

As the whole amount of the resource should be allocated, the sets of feasible decisions in stage 3 are as follows:

$$
\begin{gathered}
\mathbf{X}_{3}(0)=\{0\}, \mathbf{X}_{3}(1)=\{1\}, \mathbf{X}_{3}(2)=\{2\}, \mathbf{X}_{3}(3)=\{3\}, \\
\mathbf{X}_{3}(4)=\{4\}, \mathbf{X}_{3}(5)=\{5\}, \mathbf{X}_{3}(6)=\{6\}
\end{gathered}
$$

Finally, the only feasible state at the end of stage 3 is $y_{4}=0$. Thus:

$$
\mathbf{Y}_{4}=\{0\}
$$

The total number of process realizations is 28. First, the efficient process realizations are identified. We start from the last stage. Since only one feasible decision exists for each feasible state in this stage, all decisions in stage 3 are efficient. Next, we identify the efficient partial realizations for stage 2. We use formula (2) to calculate probabilities of reaching a particular level of achievement for each goal. Next, we use stochastic dominance rules to compare partial process realizations for each feasible state at the beginning of stage 2. A partial process realization is defined by the decisions made in stages 2 and 3 .

For the state $y_{2}=0$, there is only one feasible partial process realization: $(0,0)$, which is obviously efficient. For the state $y_{2}=1$, there are two feasible partial realizations: $(1,0)$ and $(0,1)$. According to the former, the only available unit of resource is allocated to project 2 , while according to the latter, one unit 
of resource is allocated to project 3. Table 4 presents stochastic dominance relations that occur between partial realizations $(1,0)$ and $(0,1)$.

\begin{tabular}{|c|c|c|c|c|c|c|c|c|}
\hline Goal 1 & $(0,1)$ & $(1,0)$ & Goal 2 & $(0,1)$ & $(1,0)$ & Goal 3 & $(0,1)$ & $(1,0)$ \\
\hline$(0,1)$ & $\mathrm{X}$ & & $(0,1)$ & $\mathrm{X}$ & & $(0,1)$ & $\mathrm{X}$ & \\
\hline$(1,0)$ & FSD & $\mathrm{X}$ & $(1,0)$ & FSD & $\mathrm{X}$ & $(1,0)$ & FSD & $\mathrm{X}$ \\
\hline
\end{tabular}

Table 4: Stochastic dominance relations between partial process realizations for state $y_{2}=1$.

\begin{tabular}{|c|c|c|c|c|c|c|c|c|c|}
\hline \multirow{2}{*}{$\begin{array}{c}\text { Process } \\
\text { realization }\end{array}$} & \multirow{2}{*}{ Level } & \multicolumn{3}{|c|}{ Probability } & \multirow{2}{*}{$\begin{array}{l}\text { Process } \\
\text { realization }\end{array}$} & \multirow{2}{*}{ Level } & \multicolumn{3}{|c|}{ Probability } \\
\hline & & Goal 1 & Goal 2 & Goal 3 & & & Goal 1 & Goal 2 & Goal 3 \\
\hline \multirow[t]{4}{*}{$(0,0,6)$} & 0 & 0.100 & 0.100 & 0.100 & \multirow[t]{3}{*}{$(1,2,3)$} & 3 & 0.310 & 0.324 & 0.142 \\
\hline & 1 & 0.300 & 0.200 & 0.100 & & 4 & 0.200 & 0.125 & 0.038 \\
\hline & 2 & 0.300 & 0.300 & 0.300 & & 5 & 0.060 & 0.014 & 0.004 \\
\hline & 3 & 0.300 & 0.400 & 0.500 & \multirow[t]{6}{*}{$(1,3,2)$} & 0 & 0.010 & 0.036 & 0.224 \\
\hline \multirow[t]{5}{*}{$(0,1,5)$} & 0 & 0.030 & 0.040 & 0.070 & & 1 & 0.095 & 0.177 & 0.344 \\
\hline & 1 & 0.190 & 0.180 & 0.170 & & 2 & 0.235 & 0.322 & 0.280 \\
\hline & 2 & 0.370 & 0.340 & 0.410 & & 3 & 0.355 & 0.302 & 0.116 \\
\hline & 3 & 0.270 & 0.320 & 0.290 & & 4 & 0.255 & 0.142 & 0.032 \\
\hline & 4 & 0.140 & 0.120 & 0.060 & & 5 & 0.050 & 0.021 & 0.004 \\
\hline \multirow[t]{5}{*}{$(0,3,3)$} & 0 & 0.020 & 0.090 & 0.200 & \multirow[t]{6}{*}{$(1,4,1)$} & 0 & 0.030 & 0.030 & 0.216 \\
\hline & 1 & 0.180 & 0.270 & 0.320 & & 1 & 0.140 & 0.160 & 0.366 \\
\hline & 2 & 0.360 & 0.350 & 0.300 & & 2 & 0.290 & 0.315 & 0.254 \\
\hline & 3 & 0.360 & 0.230 & 0.140 & & 3 & 0.320 & 0.305 & 0.132 \\
\hline & 4 & 0.080 & 0.060 & 0.040 & & 4 & 0.180 & 0.155 & 0.030 \\
\hline \multirow[t]{6}{*}{$(0,4,2)$} & 0 & 0.020 & 0.080 & 0.210 & & 5 & 0.040 & 0.035 & 0.002 \\
\hline & 1 & 0.090 & 0.260 & 0.340 & \multirow[t]{5}{*}{$(1,5,0)$} & 0 & 0.050 & 0.030 & 0.160 \\
\hline & 2 & 0.220 & 0.340 & 0.250 & & 1 & 0.150 & 0.130 & 0.280 \\
\hline & 3 & 0.310 & 0.230 & 0.150 & & 2 & 0.350 & 0.260 & 0.300 \\
\hline & 4 & 0.260 & 0.080 & 0.040 & & 3 & 0.350 & 0.370 & 0.220 \\
\hline & 5 & 0.100 & 0.010 & 0.010 & & 4 & 0.100 & 0.210 & 0.040 \\
\hline \multirow[t]{4}{*}{$(0,6,0)$} & 0 & 0.100 & 0.000 & 0.200 & \multirow[t]{7}{*}{$(2,2,2)$} & 0 & 0.024 & 0.036 & 0.245 \\
\hline & 1 & 0.300 & 0.200 & 0.200 & & 1 & 0.098 & 0.174 & 0.322 \\
\hline & 2 & 0.300 & 0.400 & 0.400 & & 2 & 0.223 & 0.328 & 0.268 \\
\hline & 3 & 0.300 & 0.400 & 0.200 & & 3 & 0.298 & 0.302 & 0.124 \\
\hline \multirow[t]{5}{*}{$(1,0,5)$} & 0 & 0.050 & 0.030 & 0.080 & & 4 & 0.238 & 0.136 & 0.035 \\
\hline & 1 & 0.250 & 0.160 & 0.180 & & 5 & 0.104 & 0.024 & 0.006 \\
\hline & 2 & 0.350 & 0.330 & 0.440 & & 6 & 0.015 & 0.000 & 0.000 \\
\hline & 3 & 0.250 & 0.340 & 0.260 & \multirow[t]{6}{*}{$(3,1,2)$} & 0 & 0.018 & 0.032 & 0.294 \\
\hline & 4 & 0.100 & 0.140 & 0.040 & & 1 & 0.099 & 0.152 & 0.308 \\
\hline \multirow[t]{6}{*}{$(1,1,4)$} & 0 & 0.045 & 0.024 & 0.168 & & 2 & 0.235 & 0.308 & 0.246 \\
\hline & 1 & 0.210 & 0.140 & 0.282 & & 3 & 0.331 & 0.324 & 0.114 \\
\hline & 2 & 0.335 & 0.292 & 0.300 & & 4 & 0.247 & 0.160 & 0.032 \\
\hline & 3 & 0.255 & 0.284 & 0.188 & & 5 & 0.070 & 0.024 & 0.006 \\
\hline & 4 & 0.120 & 0.176 & 0.056 & \multirow[t]{5}{*}{$(6,0,0)$} & 0 & 0.100 & 0.000 & 0.300 \\
\hline & 5 & 0.035 & 0.084 & 0.006 & & 1 & 0.300 & 0.100 & 0.300 \\
\hline \multirow[t]{3}{*}{$(1,2,3)$} & 0 & 0.030 & 0.027 & 0.200 & & 2 & 0.300 & 0.400 & 0.200 \\
\hline & 1 & 0.130 & 0.162 & 0.330 & & 3 & 0.300 & 0.500 & 0.200 \\
\hline & 2 & 0.270 & 0.348 & 0.286 & & & & & \\
\hline
\end{tabular}

Table 5: Probabilities that a particular level of goal is achieved for efficient process realizations 
As for each goal, the partial process realization $(1,0)$ dominates the partial process realization $(0,1)$, the partial realization $(0,1)$ is not efficient and according to the FSD rule. Consequently, no process realization that includes the partial realization $(0,1)$ will be considered.

In the same way, partial realizations for other feasible states at stage 2 are analyzed. Next, we go to stage 1 and compare all process realizations that comprise non-dominated partial process realizations from stage 2 by using stochastic dominance rules. Finally, the set of efficient process realizations is as follows:

$$
\begin{aligned}
& (0,0,6) ;(0,1,5) ;(0,3,3) ;(0,4,2) ;(0,6,0) ;(1,0,5) ;(1,1,4) ; \\
& (1,2,3) ;(1,3,2) ;(1,4,1) ;(1,5,0) ;(2,2,2) ;(3,1,2) ;(6,0,0) .
\end{aligned}
$$

The probabilities of reaching the successive levels of goal achievement for efficient process realizations are presented in Table 5.

Let us assume that the decision maker decided to analyze the probability that level 2 or higher will be reached for each goal. The example below provides a dialog conducted with the decision maker.

\section{Iteration 1}

The potency matrix is presented to the decision maker Table 6).

\begin{tabular}{|l|c|c|c|}
\hline & $\begin{array}{c}\text { Probability of } \\
\text { reaching at least } \\
\text { level 2- goal 1 }\end{array}$ & $\begin{array}{c}\text { Probability of } \\
\text { reaching at least } \\
\text { level 2- goal 2 }\end{array}$ & $\begin{array}{c}\text { Probability of } \\
\text { reaching at least } \\
\text { level 2- goal 3 }\end{array}$ \\
\hline Pessimistic value & 0.600 & 0.640 & 0.398 \\
\hline Optimistic value & 0.895 & 0.900 & 0.800 \\
\hline
\end{tabular}

Table 6: Potency matrix in iteration 1.

The decision maker expresses dissatisfaction with the pessimistic value for goal 3, and asks to consider only those solutions for which the probability that goal 3 will reach at least level 2 is not less than 0.5 . The following process realizations do not satisfy this constraint:

$$
(0,3,3) ;(0,4,2) ;(1,2,3) ;(1,3,2) ;(1,4,1) ;(2,2,2) ;(3,1,2) ;(6,0,0) \text {. }
$$

As a result, six process realizations are considered in the next iteration:

$$
(0,0,6) ;(0,1,5) ;(0,6,0) ;(1,0,5) ;(1,1,4) ;(1,5,0) \text {. }
$$




\section{Iteration 2}

A new potency matrix is presented to the decision maker (Table 7).

\begin{tabular}{|l|c|c|c|}
\hline & $\begin{array}{c}\text { Probability of } \\
\text { reaching at least } \\
\text { level 2- goal 1 }\end{array}$ & $\begin{array}{c}\text { Probability of } \\
\text { reaching at least } \\
\text { level 2- goal 2 }\end{array}$ & $\begin{array}{c}\text { Probability of } \\
\text { reaching at least } \\
\text { level 2- goal 3 }\end{array}$ \\
\hline Pessimistic value & 0.600 & 0.700 & 0.550 \\
\hline Optimistic value & 0.800 & 0.840 & 0.800 \\
\hline
\end{tabular}

Table 7: Potency matrix in iteration 2.

The decision maker expresses dissatisfaction with the pessimistic value for goal 1 , but is unable to specify a satisfactory probability value. The decision maker asks only to improve the pessimistic value for goal 1 . As for the two solutions $(0,0,6)$ and $(0,6,0)$, the probability of reaching at least level 2 is equal to the pessimistic value 0.6 . The solutions are excluded and the following ones are taken into account in the next iteration:

$$
(0,1,5) ;(1,0,5) ;(1,1,4) ;(1,5,0) \text {. }
$$

\section{Iteration 3}

A new potency matrix is presented to the decision maker (Table 8).

\begin{tabular}{|l|c|c|c|}
\hline & $\begin{array}{c}\text { Probability of } \\
\text { reaching at least } \\
\text { level } 2-\text { goal } 1\end{array}$ & $\begin{array}{c}\text { Probability of } \\
\text { reaching at least } \\
\text { level } 2-\text { goal } 2\end{array}$ & $\begin{array}{c}\text { Probability of } \\
\text { reaching at least } \\
\text { level } 2-\text { goal } 3\end{array}$ \\
\hline Pessimistic value & 0.700 & 0.780 & 0.550 \\
\hline Optimistic value & 0.800 & 0.840 & 0.760 \\
\hline
\end{tabular}

Table 8: Potency matrix in iteration 3.

The decision maker expresses satisfaction with pessimistic values for all goals and decides to choose the solution that maximizes the probability for goal 2 . As a result, the process realization $(1,5,0)$ is identified as the final solution.

\section{Final remarks}

The allocation problem considered in this paper can be used for describing a wide range of real-world problems. Dynamic programming is an efficient tool for solving it. However, in a multiobjective environment, it must be used together with a procedure for identifying the final solution. We propose the use of an interactive method in which preference information is obtained in a stepwise manner. This allows the decision maker to obtain better insight into trade-offs 
among different criteria. It is often pointed out that decision makers put much reliance in solutions generated through interactive procedures, and as a result, such solutions have a better chance of being implemented.

The interactive procedure presented here is a modification of the INSDECM method proposed in [7]. It was originally designed for static problems. Here, we have presented a version adapted to dynamic problems, in which not a single decision but a series of decisions were made. We have analyzed such problems in [9]. In this paper, we propose a different method. The decision maker does not have to specify the aspiration levels $\tilde{\boldsymbol{g}}_{m}^{(l)}$ in each iteration, but simply indicate the criterion that is to be improved. Thus, the amount of preference information that the decision maker has to provide is smaller.

In our problem, the decision makers defines their own requirements relative to the realization processes. However, in many dynamic problems they may wish to formulate their requirements relative to period results. Our plan for future research is to propose a procedure that enables decision makers to specify their needs in such a manner. A mixed problem should also be considered, in which some of the criteria are stochastic, while others are deterministic or fuzzy.

\section{Acknowledgement}

This research was supported by National Science Center, Decision no. DEC2013/11/B/HS4/01471.

\section{References}

[1] Bellman, R. (1957). Dynamic programming. Princeton: Princeton University Press.

[2] Bellman, R. and Dreyfus S. (1962). Applied dynamic programming. Princeton: Princeton University Press.

[3] Hariharan, L. (2009). Resource allocation problems in stochastic sequential decision making. Ph.D. Thesis. Massachusetts Institute of Technology, Department of Civil and Environmental Engineering.

[4] Johansson, R., Martenson, C., Suzić, R. and Svenson, P. (2005). Stochastic dynamic programming for resource allocation. Command and Control Systems. Swedish Defence Research Agency.

[5] Li, W. (2012) Dynamic resource allocation using stochastic optimization in wireless communications. Ph. D Thesis. The Chinese University of Hong-Kong.

[6] Mendelson, H., Pliskin, J. and Yechiali, U. (1980) A stochastic allocation problem. Operations Research, 28, 687-693. doi:10.1287/opre.28.3.687.

[7] Nowak, M. (2006). INSDECM - An interactive procedure for discrete stochastic multicriteria decision making problems. European Journal of Operational Research, 175(3), 1413-1430. doi:10.1016/j.ejor.2005.02.016. 
[8] Nowak, M. (2011). Interactive multicriteria decision aiding under risk - methods and applications. Journal of Business Economics and Management, 12, 69-91. doi:10.3846/16111699.2011.555366.

[9] Nowak, M., Trzaskalik, T. (2013) Interactive approach for a multiobjective stochastic discrete dynamic problem. Journal of Global Optimization, 57(2), 315330. doi:10.1007/s10898-012-0019-9.

[10] Trzaskalik, T. (2008) Computer-assisted introduction to operations research. Warsaw: PWE (in Polish).

[11] Trzaskalik, T. and Sitarz, S. (2002). Dynamic discrete programming with partially ordered criteria set. In: Trzaskalik, T. and Michnik, J. (Eds.). Multiple Objective Programming and Goal Programming. Recent Developments (pp. 186-195). Heidelberg: Physica-Verlag.

[12] Trzaskalik, T., Sitarz, S. (2004). Dynamic programming models in ordered structures. In: Kacprzyk, J., Słowiński, R. (eds.). Operations and System Research '04, 15-30. Warszawa: EXIT (in Polish).

[13] Trzaskalik, T., Sitarz, S. (2007). Discrete dynamic programming with outcomes in random variable structures. European Journal of Operational Research, 177(3), 1535-1548. doi:10.1016/j.ejor.2005.10.019. 